\title{
Contents, Vol. 120, 1950
}

\section{Index}

Alvarez, A., Ring Abscess of the Cornea Treated with Sulfadiazine and

Penicillin 206

Alvaro, M. E., Simultaneous Surgical Correction of Vertical and Horizontal

Deviations 191

Amsler, M., De la tectonique des greffons cornéens perforants ... 15

Discussion: Goldmann, Amsler.A Aató, S., Angiodiathermy and its Application in Glaucoma ....

325Babel, J., Une forme rare de tumeur orbitaire: le granulome éosinophile . 63Bangerter, A.,

Über plastische Operationen 83

Diskussion: Weber. Schlußwort: Bangerter.

Barth, J., Weitere Erfahrungen mit Daueriontophorese $\quad 97$

Bischler, V., vide Franeeschetti, A. Blum, J. D,, vide France schetti, A.

Bock, R. H., Zur klinischen Messung der Papillenfarbe 174

Bõhringer, H. R., und F. Verreg, Operationsresultate bei postuveitischer

Cataracta complicata; prognostische Bedeutung der diagnostischen

Vorderkammerpunktion 45

Diskussion: Bangerter. Schlußwort: Verreg.Bourquin, A., vide Dufour, R.Bruckner, A., Kurze

Mitteilungen 38

Diskussion: Goldmann. Cometta, F., E. Juillard et P. Rosselet, A propos d'un cas d'acrocéphalosyndactylie 72

Condrau, G., vide Klinger, $\mathrm{M}$.

Cuendet, J. F., et A. Moginier, Métastases choroïdennes bilatérales d'un

réticulosarcome

60

Davson, H., and A. Huber, Experimental Hypertensive Uveitis in the Rabbit 118

Discussion: Goldmann. Conclusion: Huber.Delia Casa, F., Spontanperforation der Cornea beider Bulbi .... 9

Ditrói, G., Über das Azulenol 168

Doesschate, G. ten, F. C. Donders' Last Writings 434

Doret, M., vide Franceschetti, A.

Dufour, R., et A. Bourquin, Deux cas d'uvéoparotidite histologiquement tuberculeuse 50

Discussion: Verreg. Fazakas, S., Les penicillium dans les cultures de champignons et de bactéries 418

Forni, S., Dégénérescence polymorphe familiale de la membrane

limitante postérieure de la cornée 9

Franceschetti, A., et V. Bischler, La sclérite nodulaire nécrosante et ses

rapports avec la scléromalacie 36

Franceschetti, A., et J. D. Blum, Indications et résultats de la myectomie

du petit oblique. 
Discussion: Streiff.

Franceschetti, A., et M. Doret, Kératoplastie à chaud 11

Franceschetti, A., et W. Stadlin, Hidradénomes (syringomes) des

paupières 3

Goldmann, H, Das Minutenvolumen der menschlichen Vorderkammer bei

Normalen und bei Fallen von primärem Glaukom 19, 150

Diskussion: Verreg, Huber. Schlußwort: Goldmann. Goldmann, H., H. König und F. Måder, Die

Durchlässigkeit der Augen-

linse für Infrarot $\quad 43,198$

Schlußwort: Goldmann. Grósz, S. de, Hormone Excretion of Glaucomatous Patients. Comments

on

the paper of M. Radnót and M. Csillag (Ophthalmologica Vol. 118,

998, 1949) 400

Halpern, L., Optic Coordinates' Vertigo 320

Haselmann, G., und K. Pulfrich, Acetylcholin zur Behandlung von Augen-

verätzungen 381

Hoffmann-Egg, L., Bedeutung histologischer Augenbefunde fur die Beur-

teilung perforierender Augenverletzungen 64

Diskussion: Goldmann, Huber, Verrey, Weber.

Huber, A, Zur homonymen Hemianopsie nach occipitaler Lobektomie . 70 Diskussion:

Goldmann. Schlußwort: Huber.

- $\quad$ vide Davson, $\mathrm{H}$.

Jean-Sédan, et S. Sédan-Bauby, Myopie glaucomateuse 316

Jent-Peyer, S., Zur Prognose des malignen Melanoms der Uvea ... 57

Diskussion: A. Bruckner, E. B. Streiff.

Jonkers, G. H., The Oblique Prisma 426

Juillard, E., vide Cometta, F.

Kahán, A., Diagnostische Hilfsmittel bei der AntihistamintheГapie . 391 Kettesy, A.,

Zyklanämisation, ein neues Verfahren gegen Glaukom . . 334 Klingler, M., und G. Condrau,

Lokalisatorisch irreführende Gesichtsfeld-

symptome bei Hirntumoren 270

König, H., vide Goldmann, H. Mäder, F., vide Goldmann, H.

Maeder, G., et P. Müller, Rétinite pigmentaire unilatérale 63

Moginier, A., vide Cuendet, J. F.

Mortelmans, L., Dégénéresce $\pi$ ce Maculaire Familiale (avec planche I) . 157

Müller, P., vide Maeder, G.

Neuenschwander, M., Mintacol «Bayer», ein neues Glaucom-Mittel . . 104

Diskussion: Goldmann. Schlußwort: Neuenschwander. Nordheim, R. W. von, A Modification of the Suture, Recommended by

Kalt a. o 416

PajtáS, J., Cas de pseudogliome familial héréditaire dans trois generations

(Retinitis exsudativa Coats) 411

Pascal, J. I., Basis and Applications of the Accommodative Unit . . 428 Pelláthy, B., Neuartiges

Verfahren für die Plastik des oberen Augenlids . 349 Podestà, H., Goethes Lehre von den «pathologischen Farben».. . 354 Pulfrich, K., vide Haselmann, G. Raáb, C., Die praktische Bedeutung der Gleichgewichtsstörungen der Au- 
genmuskeln 220

Rickenbach, K., und H. Werner, Scheinbarer Abflußdruck, Tension und

Druck in Kammerwasservenen 22

Rintelen, F., und H. Smolik, Über den Einfluß des Hydergins auf den

intraokularen und diastolischen Zentralarteriendruck $\quad 100$

Diskussion: Goldmann.

Rosen, E., Hemangioma of the Choroid

127

Rosselet, P., vide Cometta, F.

Rumpf, J., Petite anse pour Гextraction du cristallin 44

Saubermann, G., Penicillinkonzentrationen in Vorderkammer und Glas-

körper 27

Diskussion: Verrey. Schlagenhauff, K., Über Karzinommetastasen im Bereiche des Ciliar-

körpers. (Hierzu Tafel II.) 299

Sédan-Bauby, S., vide Jean-Sédan.

Smolik, H., vide Rintelen, F.

Stadlin, W., vide Franceschetti, A.

Steiger, M., Ein einfacher Trübungsmesser für die Praxis 109

Diskussion: Goldmann. Straub, W., Beitrag zur angeborenen Totaltrübung der Hornhaut . . . 401

Strebel, J., Über Akuleatenstichverletzungen des Auges und über die To-

leranz eines während mehr als zwanzig Jahren in Cornea-Vorderkam-

mer steckenden Bienenstachels 16

Diskussion: Verrey.

- $\quad$ Zur Vermeidung von Augenverätzungen durch Kalkmilch bei der ge-bräuchlichen

Verwendung von Baumspritzen für die Stall-Weißelung 69

- $\quad$ Über unsern Probierkasten von Plexi-Kontaktschalen

112

Streiff, E. B., Dysmorphie mandibulo-faciale (tête d'oiseau) et alterations

oculaires $\quad 79$

Verrey, F., Kératite congénitale

4

- $\quad$ vide Böhringer, H. R.

Viabec, F., Quelques details histologiques intéressants des tumeurs

«mixtes » de la glande lacrymale 210

Weekcrs, L. et R., Contribution à la pathogénie du buphtalmos . . 285 Werner, H.,

Biomikroskopische Untersuchungen über den Ansatz der hin-

tern Zonulafasern an der Linse

47

- $\quad$ vide Ríckenbach, $\mathrm{K}$.

Witmer, R., Erfahmngen mit PAS bei der Behandlung tuberkulöser

Augenleiden 106

Diskussion: Goldmann, Weber. Schlußwort: Witmer.

Zehnder-Albrecht, S., Zur Standardisierung der Perimetrie .... 255

GESELLSCHAFTSBERICHTE - SOCIETY TRANSACTIONS - SOCIÉTÉS

Schweizerische Ophthalmologische Gesellschaft. 42. GeneГalversammlungin Fribourg, 30.

September bis 2. Oktober 1949. - Société Suissed'Ophthalmologie. 42e Assemblée générale à

Fribourg, 30 Septembreau 2 octobre 1949 I

The Netherlands Ophthalmological Society. 116th Meeting held 28 and

29 May, 1949, in the Netherlands Eye Hospital, Utrecht . . 178,237

- 117th Meeting, held on Sunday, 18th September 1949, in the Wilhel- 
mina-Gasthuis, Amsterdam 365

BUCHBESPRECHUNGEN - BOOKS REVIEW - LIVRES NOUVEAUX

$125,190,253,374,437$

V. Varia 126

A He Rechte vorbehalten Cliches: Aberegg-Steiner \& Cie. AC

Bern. Buchdruckerei Friedrich Reinhardt AC

Basel 